\title{
Quality of life and wellbeing among HIV outpatients in East Africa: a multicentre observational study
}

\author{
Richard Harding ${ }^{1 *}$, Victoria Simms ${ }^{1,2}$, Suzanne Penfold ${ }^{1}$, Julia Downing ${ }^{1,3}$, Eve Namisango ${ }^{3}$, Richard A Powell ${ }^{4}$, \\ Faith Mwangi-Powell ${ }^{5}$, Scott Moreland ${ }^{6}$, Nancy Gikaara ${ }^{3}$, Mackuline Atieno ${ }^{3}$ and Irene J Higginson ${ }^{1}$
}

\begin{abstract}
Background: Global health investment has reduced HIV mortality and transmission. However, little is known of patient-reported outcomes alongside ART rollout. This study aimed to measure wellbeing using patient-reported outcome measures (PROMS) among outpatients at PEPFAR-funded facilities.

Methods: In a multicentre 2 country cross-sectional study, adults attending 12 facilities in Kenya and Uganda gave self-reported data on quality of life (physical and mental wellbeing dimensions), functional and a measure of multidimensional problems (physical, psychological, social and spiritual).

Results: Among the 1,337 participants, multidimensional problems were more common in psychological, spiritual and social domains than in physical. In multivariable analysis using GEE to adjust for facility effect, the mental health subscale of quality of life was lower for people with limited functional status $(B=-5.27,95 \% \mathrm{Cl}-5.99,1 .-4.56 p<$ $0.001)$ and higher for wealthier people $(B=0.91,95 \% C l 0.48,1.33, p<0.001)$. The physical health subscale of quality of life was lower for those with limited functional status $(B=-8.58,95 \% \mathrm{Cl}-9.46$ to $-7.70, p<0.001)$ and those who had a caregiver present $(B=-1.97,95 \% C l-3.72$ to $-0.23, p=0.027)$, higher for wealthier people $(B=1.14,95 \% C l 0.65,1.64$, $\mathrm{p}<0.001)$, and positively associated with $\mathrm{CD} 4$ count $(\mathrm{B}=1.61,95 \% \mathrm{Cl} 1.08-2.14, \mathrm{p}<0.001)$. Multidimensional problems were more burdensome for people with limited functional status $(B=-2.06,95 \% \mathrm{Cl}-2.46$ to $-1.66, p<0.001)$, and less burdensome with more education $(B=0.63,95 \% \mathrm{Cl} 0.25-1.00, p=0.001)$ or $A R T$ use $(B=0.94,95 \% C l 0.34-1.53$, $p=0.002)$.

Conclusions: Multidimensional problems are highly prevalent, and worse with declining function. Importantly, ART use does not appear to be protective for self-reported physical and mental dimensions of quality of life. Assessment and management of self-reported wellbeing must form part of HIV care and treatment services to ensure maximum benefit from ART investment.
\end{abstract}

Keywords: HIV, Quality of life, Self-report, Sub-Saharan Africa, Mental health

\section{Background}

High prevalence and associated burden of physical and psychological problems have been reported among HIV outpatients with access to antiretroviral therapy (ART) in high income countries [1-4]. Within the generalised epidemic of sub-Saharan Africa, evidence of patientreported problems since the rollout of ART has mainly

\footnotetext{
* Correspondence: richard.harding@kcl.ac.uk

'King's College London, Department of Palliative Care, Policy \&

Rehabilitation, Cicely Saunders Institute, Bessemer Road, London SE5 9RJ, UK Full list of author information is available at the end of the article
}

related to later stages of HIV disease [5,6]. The World Health Organisation (WHO) definition of health is 'complete physical, mental and social well-being', [7] and WHO guidance also identifies the need for the assessment and management of physical, psychological, social and spiritual problems from the point of HIV diagnosis and alongside treatment [8].

Although global evidence suggests that patients bear a high burden of problems from the point of HIV diagnosis [9], clinical skills and research since the advent of ART have focused mainly on outcomes of viral suppression and 
delaying mortality, and arguably have lost patientcentredness [10-12]. Patient reported outcome measures (PROMS) have been identified as essential tools to promote quality and equity in healthcare [13]. An evaluation of the effect of PEPFAR (President's Emergency Plan for AIDS Relief) funding in its target countries has established that there has been a decrease in HIVrelated deaths [14] and a reduction in the number of HIV-positive births [15]. However, there has been a lack of evaluative evidence of the management of patients' health from their own perspective; therefore it is unclear whether optimal benefit is being achieved from treatment investment.

This paper aims to measure patient wellbeing using PROMS among HIV outpatients at PEPFAR-funded facilities, and to determine associations with patient problems.

\section{Methods}

\section{Study design}

As part of a mixed-methods Public Health Evaluation (PHE) of the President's Emergency Plan for AIDS Relief (PEPFAR) Care and Support programme, a multi-centre, longitudinal quantitative study was conducted of care received and patient-health outcomes over three months in Kenya and Uganda (full protocol available [16]). This paper presents the cross-sectional analysis of results at baseline.

\section{Study design and setting}

During the longitudinal observational cohort of the PHE, consecutive HIV-infected outpatients were recruited from 12 PEFAR-funded care facilities in Kenya and Uganda. Kenya and Uganda are PEPFAR focus countries with generalised epidemics.

\section{Ethics}

Ethical approval to undertake the study was received from the Uganda National Council for Science and Technology (UNCST, Ref SS 1964), the Kenyan Medical Research Institute (Ref KEMRI/RES/7/3/1) and the College Research Ethics Committee at King's College London (Ref CREC/06/07-140). Subsequent tool changes following initial piloting were also approved.

\section{Procedure for recruitment, data collection and analysis}

Adult outpatients who were aware of their HIV diagnosis and able to give informed consent (either written or thumb print depending on literacy level) were invited into the study. Consecutive patients were approached in outpatient waiting areas in the order of their attendance, and trained researchers conducted consenting and data collection. All questionnaires were forward/back translated into local languages, and all questions read aloud. Respondents gave a verbal response to each item, and the study researcher recorded the response. This reduced any potential bias, as limited literacy could lead to a mix of self-completion and researcher-completion. Patients gave a self-report on demographic data as follows; age (coded 18-29, 30-39, 40-63), gender (male/female), country of recruitment (Kenya/Uganda), number of dependents (coded as $0-1,2-3,4+$ ), time to travel to the facility (coded as an hour or less/more than an hour), and ART use (yes/no). Socioeconomic status was measured following the methodology used in the Demographic and Health Surveys [17], using variables including house construction, possession of items, fuel supply etc. The entire sample of 1,337 people was included. ART use and CD4 count were the only variables not self-reported (these were extracted from file).

Finally three self-report scales were completed, each having been previously validated in sub-Saharan African populations. These were: the Medical Outcome Scale HIV (MOS-HIV), which is a 35-item quality of life scale in Uganda and widely used in Africa $[18,19]$ consists of two subscales of physical and mental health (each subscale analysed as quintiles) and is sensitive to treatment effects [20]; the APCA African Palliative Outcome Scale (POS) [21] has seven patient-reported multidimensional items that reflects the WHO definition of palliative care, (i.e. it measures physical, psychological, social and spiritual problems among those with incurable life-limiting illness, analysed as quintiles, and referred to as the POS); the ECOG Performance Status, which is a single item rating of functional status ranging from $0=$ fully active to 5 =dead. $($ coded $0 / 1 / 2+)$ [22]. Participants were paid $\$ 5$ for travel expenses.

\section{Data management and entry}

Data were collected in a private space away from other patients, and questionnaires carried only a unique study participant identifier. Questionnaires were stored in a locked cabinet. Data were entered into a pre-designed EpiData v3.1 database (Odense Denmark: EpiData Association; 2000-2008). Errors were identified using consistency checks and double-entry validation, and followed-up by manual checking of questionnaires. Stata v10 (Texas: Stata Corp LP; 2007) was used to undertake descriptive analysis and tests of association. MOS-HIV item scores were converted into continuous summary scores for mental and physical health (0-100) [23]. The scores for the seven patient-completed items of the APCA African POS (pain, symptoms, worry, peace/spiritual wellbeing, sharing feelings, feeling life worthwhile, help and advice) were summed to create a total patient POS score. Additionally, all individual POS item scores were coded into ternary variables $(0 / 1=$ low problem, $2 / 3=$ moderate problem, $4 / 5=$ severe problem) presented by facility. The wealth quintile variable 
was created following the methods of the Demographic Health Surveys $[17,24]$, using factor analysis with principal components analysis to create a continuous variable. This variable was then divided into quintiles. Age was grouped into categories: $18-29,30-39,40-49,50-$ 59 and 60-70. CD4 was categorised 0-100, 101-200, 201-350 and 351 to maximum. Demographic characteristics and outcomes were described by facility (facilities were labelled A-M to retain anonymity). The association between continuous outcomes (mental health, physical health and total POS score) and facility was tested using analysis of variance.

The main outcomes were quality of life using two subscales of physical and mental health score from the MOS-HIV, and the POS [23].

T-tests and analysis of variance were used to identify demographic and clinical characteristics associated with the three outcomes in bivariate analysis. The individual POS item scores were included as co-variates for quality of life mental and physical health subscales but not for total POS scores, because they were components of it and so association was inevitable. Multilevel fixed-effects linear regression was used to analyse whether scores were associated with personal characteristics, adjusting for facility clustering. All variables associated with an outcome in bivariate analysis were taken forward into the multivariate model. CD4 count was not used in a model if the presence of a CD4 test result on file was associated with the outcome. Population-averaged models were fitted using generalised estimating equations (GEE) to adjust for the effect of facility. The three outcomes were mental health subscale score, physical health subscale score, and total patient POS score.

\section{Results}

\section{Sample description}

Table 1 shows the demographic characteristics of the sample by facility. One participant did not give demographic data, leaving a total sample in the analysis of 1336 participants, an average of 111.4 per facility (range 102-125). In total $68.3 \%$ were female $(57.7 \%-78.5 \%$ by facility), and the mean age was 34.8 (32.1-38.8 by facility).

\section{Descriptive outcomes}

For the entire sample of $n=1336$, mean quality of life mental health subscale score was 46.2 (95\% CIs 45.7-46.8) (Table 2). By facility, the range was from 40.3 to 49.7 and the difference between facilities was statistically significant $(\mathrm{F}=10.46, \mathrm{p}<0.001)$. Quality of life physical health subscale mean score was 44.9 for the entire sample (95\% CIs 44.2-45.5), ranging from 37.9 to 49.5 by facility, which was statistically significant $(\mathrm{F}=12.61, \mathrm{p}<0.001)$. The mean total POS score was 20.8 (95\% CIs 20.5-21.1) and ranged from 19.1 to 22.6 by facility, which was also statistically significant $(\mathrm{F}=6.94, \mathrm{p}<0.001)$.

Review of the POS scores shows that items for which the greatest proportion of respondents fell into the "severe problem" category were 'need for help/advice' (51.6\% severe) and 'difficulty sharing feelings' (43.5\% severe) and feeling at peace (21.1\%). In seven facilities, more than half of participants had severe problems with obtaining help/advice. For both these items there was only one facility where fewer than $10 \%$ had severe problems - facility G. Psychological, spiritual and social problems were more common than physical problems.

\section{Bivariate analysis}

At 5\% significance, quality of life mental health subscale was associated with wealth quintile, education, having a caregiver present, functional status and having a CD4 count on file, while physical health was associated with age group, wealth quintile, education, ART use, CD4 count result, being newly diagnosed, having a carer present and functional status (Table 3). Poorer and less educated people, with limited functional status, those with a carer present and those lacking a CD4 count on file were more likely to have lower (worse) mental health subscale score. Older, poorer and less educated people taking ART, with a long-standing HIV diagnosis, those having a carer present and those with limited function were more likely to have lower (worse) physical health. Both the MOS-HIV outcomes were associated with CD4 count result, but since having a CD4 count was associated with mental health, the CD4 test result could not be considered as a covariate of mental health because of sampling bias. Total patient POS score was associated with wealth quintile, education, ART use, functional status and having a CD4 count on file. POS multidimensional problems were more common among people who were poorer, less educated, with lower function, who were not taking ART or did not have a CD4 count on file.

All seven patient-completed POS items were associated with both the MOS-HIV outcomes. Mental and physical health quality of life subscale scores were lower (worse) for participants with moderate or severe pain, symptoms and worry, and for those who reported low scores for finding peace or for obtaining help and advice. Participants with moderate problems finding life worthwhile had slightly lower physical and mental health scores than those with severe difficulty. Participants with severe difficulty sharing feelings had better MOS-HIV scores than those with low or moderate problems.

\section{Multivariable analysis}

Using GEE (Table 4), mental health quality of life subscale was lower (worse) for people with limited 
Table 1 Demographic characteristics of sample by facility $N=1336$

\begin{tabular}{|c|c|c|c|c|c|c|c|c|c|c|c|c|c|}
\hline & & \multicolumn{12}{|l|}{ Facility } \\
\hline & & $A$ & B & $\mathrm{C}$ & D & $\mathrm{E}$ & $\mathbf{F}$ & G & $\mathbf{H}$ & $J$ & $\mathrm{~K}$ & $\mathrm{~L}$ & $M$ \\
\hline \multicolumn{2}{|c|}{$N$} & 109 & 111 & 120 & 120 & 125 & 111 & 107 & 105 & 112 & 107 & 102 & 107 \\
\hline \multicolumn{2}{|c|}{ Gender (\% female) } & 68.8 & 74.8 & 65.8 & 72.5 & 70.4 & 57.7 & 78.5 & 64.8 & 60.7 & 61.7 & 71.6 & 72.0 \\
\hline \multicolumn{2}{|c|}{ Mean age (range) } & $33.0(18-69)$ & $38.6(21-63)$ & $33.9(18-59)$ & $33.5(18-58)$ & $34.8(19-58)$ & $33.7(18-60)$ & $37.8(18-70)$ & $38.8(21-66)$ & $32.1(18-53)$ & $33.7(22-67)$ & $34.9(18-55)$ & $33.0(19-61)$ \\
\hline \multirow[t]{5}{*}{ Education } & None & 5.6 & 2.7 & 1.7 & 5.0 & 2.4 & 0.9 & 25.2 & 14.3 & 4.5 & 3.7 & 6.9 & 8.4 \\
\hline & Began primary & 69.4 & 54.1 & 55.8 & 50.0 & 40.8 & 54.1 & 53.3 & 57.1 & 35.7 & 58.0 & 37.3 & 53.3 \\
\hline & $\begin{array}{l}\text { Began } \\
\text { secondary }\end{array}$ & 22.2 & 38.7 & 30.8 & 37.5 & 46.4 & 37.8 & 20.6 & 25.7 & 48.2 & 26.2 & 39.2 & 27.1 \\
\hline & Diploma & 1.9 & 3.6 & 10.8 & 7.5 & 7.2 & 6.3 & 0.9 & 1.0 & 6.3 & 10.3 & 13.7 & 8.4 \\
\hline & Degree & 0.9 & 0.9 & 0.8 & 0 & 3.2 & 0.9 & 0 & 1.9 & 5.4 & 1.9 & 2.9 & 2.8 \\
\hline \multirow[t]{5}{*}{ Wealth quintile } & Poorest & 39.5 & 2.7 & 5.0 & 1.7 & 15.2 & 37.3 & 80.4 & 43.8 & 2.7 & 3.7 & 5.9 & 19.6 \\
\hline & Middle poor & 32.1 & 27.0 & 25.8 & 3.3 & 14.4 & 24.6 & 6.5 & 47.6 & 4.5 & 10.3 & 8.8 & 26.2 \\
\hline & Middle & 19.3 & 33.3 & 15.8 & 15.0 & 33.6 & 19.1 & 10.3 & 4.8 & 19.6 & 23.4 & 15.7 & 27.1 \\
\hline & $\begin{array}{l}\text { Middle } \\
\text { wealthy }\end{array}$ & 8.3 & 19.8 & 21.7 & 28.3 & 21.6 & 12.7 & 1.9 & 2.9 & 42.9 & 30.8 & 30.4 & 17.8 \\
\hline & Wealthiest & 0.9 & 17.1 & 31.7 & 51.7 & 15.2 & 6.4 & 0.9 & 1.0 & 30.4 & 31.8 & 39.2 & 9.4 \\
\hline \multicolumn{2}{|c|}{$\begin{array}{l}\text { Has a CD4 test result on } \\
\text { file (\%) }\end{array}$} & 88.1 & 91.0 & 85.8 & 99.2 & 90.4 & 89.2 & 100.0 & 41.0 & 50.4 & 46.7 & 90.2 & 33.6 \\
\hline \multirow[t]{5}{*}{ CD4 count (\%) } & $\mathrm{N}$ & 96 & 101 & 103 & 116 & 113 & 99 & 107 & 43 & 57 & 50 & 92 & 36 \\
\hline & $0-100$ & 16.7 & 2.0 & 29.1 & 27.6 & 13.3 & 15.2 & 9.4 & 9.3 & 12.3 & 16.0 & 20.7 & 8.3 \\
\hline & $101-200$ & 15.6 & 19.8 & 19.4 & 19.8 & 17.7 & 24.2 & 30.8 & 7.0 & 10.5 & 20.0 & 16.3 & 13.9 \\
\hline & $201-350$ & 28.1 & 37.6 & 20.4 & 21.6 & 29.2 & 21.2 & 36.5 & 30.2 & 22.8 & 36.0 & 22.8 & 36.1 \\
\hline & $351+$ & 39.6 & 40.6 & 31.1 & 31.0 & 39.8 & 39.4 & 23.4 & 53.5 & 54.4 & 28.0 & 40.2 & 41.7 \\
\hline \multicolumn{2}{|c|}{ Newly diagnosed (\%) } & 36.7 & 2.7 & 63.3 & 54.2 & 19.2 & 35.1 & 0 & 17.1 & 70.8 & 25.2 & 34.3 & 29.0 \\
\hline \multicolumn{2}{|l|}{ Taking ART (\%) } & 66.7 & 67.6 & 8.3 & 40.0 & 56.5 & 31.5 & 91.6 & 36.2 & 8.9 & 14.0 & 38.2 & 60.7 \\
\hline \multicolumn{2}{|l|}{ Has a carer (\%) } & 8.3 & 9.0 & 15.8 & 12.5 & 14.4 & 10.8 & 98.1 & 3.8 & 13.4 & 3.7 & 19.7 & 9.4 \\
\hline \multirow[t]{4}{*}{ ECOG } & 0 & 56.0 & 74.8 & 80.0 & 75.0 & 58.4 & 71.2 & 20.6 & 79.1 & 82.1 & 51.4 & 43.1 & 23.4 \\
\hline & 1 & 33.9 & 22.5 & 19.2 & 20.8 & 34.4 & 20.7 & 37.4 & 19.1 & 9.8 & 43.9 & 45.1 & 63.6 \\
\hline & 2 & 9.2 & 2.7 & 0.8 & 4.2 & 6.4 & 7.2 & 38.3 & 0 & 4.5 & 2.8 & 8.8 & 8.4 \\
\hline & 3 or 4 & 0.9 & 0 & 0 & 0 & 0.8 & 0.9 & 3.7 & 1.9 & 3.6 & 1.9 & 2.9 & 4.7 \\
\hline \multicolumn{2}{|c|}{$\begin{array}{l}\text { Mean number of care } \\
\text { components received during } \\
\text { study (SD) }\end{array}$} & $15.5(6.0)$ & $11.5(6.2)$ & $8.4(4.9)$ & $15.8(6.7)$ & $10.1(5.8)$ & $9.9(5.1)$ & $23.9(4.2)$ & $22.0(6.3)$ & $13.0(6.4)$ & $13.8(3.9)$ & $20.1(4.1)$ & $16.0(4.3)$ \\
\hline
\end{tabular}


Table 2 Patient outcome scores by facility, MOS-HIV and POS N = 1336

\begin{tabular}{|c|c|c|c|c|c|c|c|c|c|c|c|c|c|c|}
\hline & & A & B & C & D & $\mathrm{E}$ & $\mathrm{F}$ & G & $\mathbf{H}$ & J & K & $\mathbf{L}$ & M & All \\
\hline \multicolumn{2}{|l|}{ Mean mental health score (SD) } & $47.0(10.1)$ & $49.7(8.2)$ & $47.5(10.2)$ & $48.4(9.5)$ & $49.5(9.7)$ & $46.4(8.6)$ & $42.6(7.3)$ & $40.3(8.9)$ & 43.9 (10.6) & $47.0(9.4)$ & $47.7(9.3)$ & $43.9(9.6)$ & $46.2(9.7)$ \\
\hline \multicolumn{2}{|l|}{ Mean physical health score (SD) } & $43.1(12.8)$ & $49.5(9.2)$ & $47.4(11.6)$ & $46.7(11.8)$ & $47.1(9.6)$ & $42.6(11.2)$ & $37.9(9.7)$ & $38.5(12.3)$ & $49.0(11.4)$ & $45.8(10.9)$ & $46.7(12.5)$ & $42.8(10.7)$ & 44.9 (11.7) \\
\hline \multicolumn{2}{|l|}{ POS: Mean total patient score (SD) } & $22.6(5.5)$ & $22.1(4.9)$ & $20.9(5.5)$ & $22.4(4.9)$ & $20.6(5.1)$ & $22.0(5.8)$ & $21.3(4.9)$ & $19.2(4.4)$ & $19.7(4.3)$ & $20.0(5.5)$ & $19.6(4.5)$ & $19.1(5.3)$ & $20.8(5.2)$ \\
\hline \multirow[t]{2}{*}{ Pain (\%) } & Moderate & 47.7 & 43.2 & 35.8 & 31.7 & 53.6 & 47.8 & 71.0 & 68.6 & 33.9 & 38.3 & 51.0 & 59.8 & 48.2 \\
\hline & Severe & 8.3 & 6.3 & 4.2 & 8.3 & 14.4 & 6.3 & 15.9 & 18.1 & 2.7 & 14.0 & 2.9 & 15.0 & 9.7 \\
\hline \multirow[t]{2}{*}{ Symptoms } & Moderate & 36.7 & 26.1 & 40.8 & 20.8 & 33.6 & 35.1 & 58.9 & 50.4 & 35.7 & 48.6 & 36.3 & 36.5 & 38.1 \\
\hline & Severe & 4.6 & 1.8 & 0.8 & 3.3 & 4.0 & 1.8 & 9.4 & 4.8 & 0.9 & 9.4 & 2.0 & 8.4 & 4.2 \\
\hline \multirow[t]{2}{*}{ Worry } & Moderate & 22.9 & 20.7 & 30.0 & 29.2 & 30.4 & 32.4 & 57.0 & 61.0 & 42.9 & 16.8 & 42.2 & 35.5 & 34.9 \\
\hline & Severe & 17.4 & 9.0 & 10.0 & 11.7 & 9.6 & 9.9 & 15.0 & 16.2 & 13.4 & 24.3 & 6.9 & 14.0 & 13.0 \\
\hline \multirow[t]{2}{*}{ Difficulty sharing feelings } & Moderate & 31.2 & 27.0 & 24.2 & 19.2 & 21.6 & 21.6 & 31.8 & 32.4 & 32.1 & 27.1 & 65.7 & 33.6 & 30.2 \\
\hline & Severe & 47.7 & 49.6 & 66.7 & 60.8 & 55.2 & 45.1 & 6.5 & 15.2 & 61.6 & 40.2 & 33.3 & 31.8 & 43.5 \\
\hline \multirow[t]{2}{*}{ Difficulty finding life worthwhile } & Moderate & 15.6 & 14.4 & 20.0 & 12.5 & 16.8 & 13.5 & 23.4 & 59.1 & 31.3 & 20.6 & 36.3 & 43.0 & 25.1 \\
\hline & Severe & 3.7 & 23.4 & 10.0 & 9.2 & 12.8 & 21.6 & 0.9 & 17.1 & 11.6 & 6.5 & 7.8 & 22.4 & 12.3 \\
\hline \multirow[t]{2}{*}{ Lack of peace } & Moderate & 28.4 & 21.6 & 30.8 & 30.0 & 28.0 & 22.5 & 42.1 & 62.9 & 48.2 & 21.5 & 49.0 & 45.8 & 35.6 \\
\hline & Severe & 11.0 & 13.5 & 15.8 & 13.3 & 26.4 & 13.5 & 33.6 & 23.8 & 23.2 & 33.6 & 11.8 & 34.6 & 21.1 \\
\hline \multirow[t]{2}{*}{ Need for help/advice } & Moderate & 22.9 & 22.5 & 14.2 & 17.5 & 27.2 & 47.8 & 48.6 & 31.4 & 14.3 & 21.5 & 32.4 & 34.6 & 27.7 \\
\hline & Severe & 36.7 & 59.5 & 69.2 & 52.5 & 51.2 & 36.9 & 10.3 & 41.9 & 77.7 & 71.0 & 65.7 & 44.9 & 51.6 \\
\hline
\end{tabular}


Table 3 Bivariate analysis for each outcome MOS-HIV physical health, MOS-HIV mental health, and POS N=1336

\begin{tabular}{|c|c|c|c|c|c|c|c|}
\hline & & \multicolumn{2}{|c|}{ Mental health score } & \multicolumn{2}{|c|}{ Physical health score } & \multicolumn{2}{|c|}{ Total patient POS score } \\
\hline & & Mean (95\% Cls) & Test result & Mean (95\% Cls) & Test result & Mean (95\% Cls) & Test result \\
\hline \multirow[t]{2}{*}{ Gender } & Male & $46.6(45.7-47.5)$ & $t=0.94, p=0.347$ & $44.1(42.9-45.2)$ & $t=-1.64, p=0.101$ & $21.0(20.5-21.6)$ & $F=1.15, p=0.284$ \\
\hline & Female & $46.1(45.4-46.7)$ & & $45.2(44.5-46.0)$ & & $20.7(20.4-21.0)$ & \\
\hline \multirow[t]{5}{*}{ Age group } & $18-29$ & $46.3(45.2-47.3)$ & $F=1.11, p=0.348$ & $45.9(44.7-47.1)$ & $F=2.64, p=0.032$ & $20.7(20.2-21.2)$ & $F=1.71, p=0.146$ \\
\hline & $30-39$ & $45.9(45.1-46.7)$ & & $45.1(44.2-46.1)$ & & $20.5(20.1-20.9)$ & \\
\hline & $40-49$ & $46.4(45.3-47.5)$ & & $43.3(41.8-44.7)$ & & $21.3(20.7-21.9)$ & \\
\hline & $50-59$ & $48.2(46.4-50.1)$ & & $43.7(41.1-46.2)$ & & $21.6(20.6-22.5)$ & \\
\hline & $60-70$ & $44.5(40.3-48.7)$ & & $42.0(39.1-44.9)$ & & $22.1(19.0-25.2)$ & \\
\hline \multirow[t]{5}{*}{ Wealth quintile } & Poorest & $43.1(42.1-44.2)$ & $F=14.41, p<0.001$ & $40.2(38.8041 .5)$ & $F=25.26, p<0.001$ & $20.8(20.1-21.4)$ & $F=2.48, p=0.042$ \\
\hline & Middle poor & $45.3(44.1-46.6)$ & & $42.9(41.5-44.4)$ & & $20.4(19.8-21.0)$ & \\
\hline & Middle & $46.4(45.2-47.5)$ & & $45.2(43.8-46.5)$ & & $20.6(19.9-21.2)$ & \\
\hline & Middle wealthy & $47.7(46.6-48.8)$ & & $47.6(46.3-48.8)$ & & $20.6(20.0-21.2)$ & \\
\hline & Wealthiest & $48.8(47.6-50.0)$ & & $48.7(47.3-50.1)$ & & $21.7(21.1-22.3)$ & \\
\hline \multirow[t]{4}{*}{ Education } & None & $43.6(41.6-45.5)$ & $F=4.65, p=0.003$ & $41.0(38.5-43.5)$ & $F=5.65, p<0.001$ & $19.6(18.5-20.7)$ & $F=5.31, p=0.001$ \\
\hline & Began primary & $46.1(45.4-46.9)$ & & $44.4(43.5-45.2)$ & & $20.7(20.3-21.0)$ & \\
\hline & Began secondary & $46.3(45.4-47.2)$ & & $45.9(44.9-46.9)$ & & $20.9(20.4-21.4)$ & \\
\hline & Diploma+ & $48.7(47.1-50.3)$ & & $46.7(44.2-49.1)$ & & $22.4(21.4-23.3)$ & \\
\hline \multirow[t]{2}{*}{ ART } & Yes & $46.4(45.7-47.2)$ & $t=-0.62, p=0.536$ & $44.0(44.7-46.4)$ & $\mathrm{t}=2.34, \mathrm{p}=0.020$ & $21.3(20.9-21.8)$ & $F=10.59, p=0.001$ \\
\hline & No & $46.1(45.4-46.8)$ & & $45.5(43.1-44.9)$ & & $20.4(20.0-20.8)$ & \\
\hline \multirow[t]{2}{*}{ Newly diagnosed } & Yes & $46.4(45.5-47.3)$ & $t=-0.46, p=0.648$ & $46.3(45.1-47.4)$ & $t=-3.09, p=0.002$ & $20.8(20.4-21.3)$ & $F=0.01, p=0.936$ \\
\hline & No & $46.2(45.5-46.8)$ & & $44.2(43.4-44.9)$ & & $20.8(20.5-21.1)$ & \\
\hline \multirow[t]{2}{*}{ Has a carer } & Yes & $43.5(42.4-44.7)$ & $\mathrm{t}=4.82, \mathrm{p}<0.001$ & $39.9(38.3-41.4)$ & $t=7.48, p<0.001$ & $21.2(20.5-21.8)$ & $F=1.50, p=0.220$ \\
\hline & No & $46.8(46.3-47.4)$ & & $46.0(45.3-46.6)$ & & $20.7(20.4-21.0)$ & \\
\hline \multirow[t]{4}{*}{ Functional status } & 0 & $49.0(48.4-49.7)$ & $F=72.73, p<0.001$ & $49.7(49.0-50.4)$ & $F=197.63, p<0.001$ & $21.8(21.5-22.2)$ & $F=32.01, p<0.001$ \\
\hline & 1 & $43.1(42.2-43.9)$ & & $39.8(38.8-40.8)$ & & $19.6(19.1-20.1)$ & \\
\hline & 2 & $39.4(38.0-40.9)$ & & $31.7(30.0-33.5)$ & & $18.1(17.1-19.1)$ & \\
\hline & $3 / 4$ & $35.7(33.2-38.2)$ & & $24.3(20.9-27.7)$ & & $17.7(15.7-19.7)$ & \\
\hline \multirow[t]{2}{*}{ Has a CD4 test result on file } & Yes & $46.9(46.3-47.4)$ & $t=-4.16, p<0.001$ & $45.1(44.4-45.8)$ & $t=-1.41, p=0.159$ & $21.0(20.7-21.3)$ & $F=7.10, p=0.008$ \\
\hline & No & $44.3(43.2-45.4)$ & & $44.1(42.7-45.4)$ & & $20.1(19.6-20.7)$ & \\
\hline \multirow[t]{4}{*}{ CD4 count } & $0-100$ & $49.0(48.2-49.9)$ & $F=8.33, p<0.001$ & $44.5(43.3-45.6)$ & $F=25.41, p<0.001$ & $20.7(19.9-21.5)$ & $F=0.38, p=0.769$ \\
\hline & $101-200$ & $50.4(49.8-51.1)$ & & $47.6(46.8-48.4)$ & & $21.2(20.4-21.9)$ & \\
\hline & 201-350 & $51.4(50.8-51.9)$ & & $49.8(49.2-50.4)$ & & $21.2(20.6-21.8)$ & \\
\hline & 351-max & $51.3(50.9-51.8)$ & & $50.5(50.0-51.0)$ & & $21.0(20.5-21.5)$ & \\
\hline
\end{tabular}


Table 3 Bivariate analysis for each outcome MOS-HIV physical health, MOS-HIV mental health, and POS $\mathbf{N}=1336$ (Continued)

\begin{tabular}{|c|c|c|c|c|c|}
\hline \multirow[t]{3}{*}{ Pain } & Low & $50.1(49.4-50.8)$ & $F=99.40, p<0.001$ & $51.3(50.5-52.1)$ & $F=217.20, p<0.001$ \\
\hline & Moderate & $44.2(43.5-44.9)$ & & $41.4(40.5-42.2)$ & \\
\hline & Severe & $39.8(38.0-41.6)$ & & $34.4(32.6-36.3)$ & \\
\hline \multirow[t]{3}{*}{ Symptoms } & Low & $48.8(48.2-49.5)$ & $F=78.98, p<0.001$ & $49.2(48.4-49.9)$ & $F=165.51, p<0.001$ \\
\hline & Moderate & $43.2(42.4-43.9)$ & & $39.7(38.8-40.7)$ & \\
\hline & Severe & $38.6(36.0-41.2)$ & & $32.3(29.5-35.1)$ & \\
\hline \multirow[t]{3}{*}{ Worry } & Low & $49.9(49.2-50.5)$ & $F=136.9, p<0.0016$ & $47.9(47.1-48.6)$ & $F=54.81, p<0.001$ \\
\hline & Moderate & $43.5(42.7-44.3)$ & & $42.4(41.3-43.5)$ & \\
\hline & Severe & $39.1(37.6-40.7)$ & & 39.6 (37.9-41.3) & \\
\hline \multirow[t]{3}{*}{ Sharing feelings } & Low & $45.4(44.4-46.4)$ & $F=6.10, p=0.002$ & $41.9(40.7-43.2)$ & $F=41.51, p<0.001$ \\
\hline & Moderate & $45.5(44.6-46.4)$ & & $42.8(41.6-43.9)$ & \\
\hline & Severe & $47.3(46.5-48.1)$ & & $48.1(47.2-48.9)$ & \\
\hline \multirow[t]{3}{*}{ Finds life worthwhile } & Low & $48.1(47.5-48.8)$ & $F=49.89, p<0.001$ & $46.0(45.2-46.8)$ & $F=12.06, p<0.001$ \\
\hline & Moderate & $42.3(41.3-43.2)$ & & $42.3(41.1-43.5)$ & \\
\hline & Severe & $44.6(43.0-46.2)$ & & $44.3(42.4-46.1)$ & \\
\hline \multirow[t]{3}{*}{ Peace } & Low & $51.0(50.3-51.7)$ & $F=169.79, p<0.001$ & $48.1(47.2-49.0)$ & $F=49.65, p<0.001$ \\
\hline & Moderate & $43.9(43.1-44.7)$ & & $43.6(42.5-44.6)$ & \\
\hline & Severe & $40.3(39.2-41.4)$ & & $40.3(38.9-41.7)$ & \\
\hline \multirow[t]{3}{*}{ Help and advice } & Low & $48.6(47.5-49.7)$ & $F=10.27, p<0.001$ & $45.3(43.9-46.7)$ & $F=5.30, p=0.005$ \\
\hline & Moderate & $45.8(44.8-46.7)$ & & $43.2(42.0-44.4)$ & \\
\hline & Severe & $45.6(44.8-46.3)$ & & $45.6(44.7-46.4)$ & \\
\hline
\end{tabular}


Table 4 Generalised estimating equations for each model MOS-HIV physical health, MOS-HIV mental health, and POS $\mathrm{N}=1336$

\begin{tabular}{|c|c|c|c|c|c|c|}
\hline & \multicolumn{2}{|l|}{ Mental health score } & \multicolumn{2}{|l|}{ Physical health score } & \multicolumn{2}{|l|}{ POS score } \\
\hline & Coefficient (95\% Cls) & $p$ & Coefficient (95\% Cls) & $p$ & Coefficient (95\% Cls) & $p$ \\
\hline Age & & & $-0.05(-1.08,0.01)$ & 0.079 & & \\
\hline Wealth quintile & $0.91(0.48,1.33)$ & $<0.001$ & $1.14(0.65,1.64)$ & $<0.001$ & $0.16(-0.08,0.39)$ & 0.192 \\
\hline Functional status & $-5.27(-5.99,-4.56)$ & $<0.001$ & $-8.58(-9.46,-7.70)$ & $<0.001$ & $-2.06(-2.46,-1.66)$ & $<0.001$ \\
\hline Newly diagnosed & & & $-0.08(-1.47,1.30)$ & 0.907 & & \\
\hline Has a carer & $-1.13(-2.63,0.37)$ & 0.141 & $-1.97(-3.72,-0.23)$ & 0.027 & & \\
\hline CD4 count & & & $1.61(1.08,2.14)$ & $<0.001$ & & \\
\hline Education & $0.07(-0.60,0.74)$ & 0.838 & $0.11(-0.69,0.92)$ & 0.783 & $0.63(0.25,1.00)$ & 0.001 \\
\hline Using ART & & & $1.00(-0.29,2.23)$ & 0.128 & $0.94(0.34,1.53)$ & 0.002 \\
\hline
\end{tabular}

functional status $(\mathrm{B}=-5.2795 \% \mathrm{CI}-5.99,-4.56)$ and slightly higher (better) for wealthier people $(B=0.91$, $95 \%$ CI 0.48 to $1.33, \mathrm{p}<0.001)$. Physical health quality of life subscale was lower (worse) for those with limited functional status $(\mathrm{B}=-8.58,95 \% \mathrm{CI}-9.46$ to -7.670 , $\mathrm{p}<0.001)$ and for those who had a caregiver present $(\mathrm{B}=-1.97,95 \% \mathrm{CI}-3.72$ to $-0.23, \mathrm{p}=0.027)$, higher (better) for wealthier people $(\mathrm{B}=1.14,95 \% \mathrm{CI} 0.65$ to $1.64, \mathrm{p}<0.001)$, and positively associated with CD4 count $(\mathrm{B}=1.61,95 \% \mathrm{CI} 1.08-2.14, \mathrm{p}<0.001)$. Multidimensional POS problems were more burdensome for people with limited functional status $(B=-2.06,95 \%$ $\mathrm{CI}-2.46$ to $-1.66, \mathrm{p}<0.001)$, less burdensome for those with more education $(\mathrm{B}=0.63,95 \%$ CI $0.25-1.00, \mathrm{p}=$ $0.001)$ or those using ART $(B=0.94,95 \%$ CI $0.34-1.53$, $\mathrm{p}=0.002)$.

\section{Discussion and conclusion}

Our data reveal severe problems for patients' access to help and advice and to their ability to share feelings. Generally, social and spiritual problems were selfreported as worse than physical problems. This suggests that the concept of health as defined by the WHO needs greater clinical attention. In the context of a generalised epidemic and limited resources, implying a high patient load per health professional, time may be limited for help and advice. Routine assessment of multi-dimensional problems and referral to non-medical/nursing staff may be a feasible approach to achieving wellbeing. Holistic assessment and care are required, especially as we found that those with worse physical and mental health reported poor wellbeing in terms of being at peace (a measure of spiritual wellbeing [25]), and gaining help and advice. It is also important to note that within the POS, the greatest proportion of respondents who identified severe problems were for the items on accessing help and advice, sharing feelings, and being at peace. Inequalities persist in terms of those with less education having a worse POS score (multidimensional problems) and worse mental health for the less wealthy, demonstrating that within the global health debate we must recognise inequalities within low income countries. The quantitative POS data are supported by qualitative data from these facilities, which identified the multidimensional problems of outpatients in line with the WHO definition of palliative care, i.e. problems in the physical, psychological, social and spiritual domains [26]. The data also demonstrate a higher burden of physical and mental ill-health, and multidimensional problems, with poorer functional status. Socioeconomic status and education are closely associated with each other, therefore only one or the other is associated with outcomes in multivariate models. We note from previous analysis of people newly diagnosed with HIV in East Africa that help/ advice is closely associated with education (contributing significantly to the total POS score) [27], which may explain our finding of an association between education rather than poverty and total POS score.

An analysis of ART-naïve HIV outpatients in Uganda found very similar MOS-HIV mean physical subscale score (46.18 vs 44.9 in our sample) and mental subscale score (46.19 vs 46.2) [28]. The MOS-HIV subscale mean scores in our sample are worse compared to recent data from Belgium (mean physical health subscale in Belgium 55.6 vs 44.9 in our sample, mean mental health 52.0 in Belgium vs 46.2 in our sample) [29] although a greater proportion of the sample in Belgium were male $(78.9 \%$ vs $31.7 \%$ and on ART (92.0\% vs $43.4 \%$ ).

There are several limitations to our data. Firstly, the use of self-report data may be less robust in regions with limited literacy and less experience of questionnaire use. We reduced this potential bias by only using questionnaires validated on local populations, and by researchers administering all questionnaires. Second, clinical data (i.e. ART use and CD4 count) were reliant on availability on file, and therefore we were unable to analyse variables where this was not routinely collected and recorded. We have reported separate analyses of factors associated with the presence of a CD4 count on file [30]. Third, the 
data are cross sectional and therefore we can determine associations not causality.

It is notable that our findings confirm previous European evidence that ART use is not associated with physical or mental health [3]. We do not suggest that ART is not effective in improving outcomes, but that people on treatment continue to have multidimensional problems that require support across domains of need to achieve optimal health. We recently completed two trails of an intervention [31] in response to the findings in the present paper that these problems persist and are burdensome alongside treatment.

From these data we conclude that all patients require holistic assessment and care irrespective of ART use or functional status, but that those with worse function or with a lower socioeconomic status require additional support beyond the medical management of their HIV disease.

\section{Competing interests}

The authors declare that they have no competing interests.

\section{Authors' contributions}

RH and IJH designed the study, VS SP led the analysis, VS SP JD EN led the study implementation and quality control, MA NG RP FMP led fieldwork, SM was consultant throughout the study. All authors were members of the Project Steering Group and advised on each stage of the study and its interpretation and reporting. All authors read and approved the manuscript.

\section{Acknowledgements}

We are grateful to the United States Agency for International Development for funding this study under a sub-agreement GPO-A-00-03-00003-00, made under the authority provided to the University of North Carolina. The present study benefited from the participation of a wide range of partners, medical professionals, HIV specialists and palliative care researchers. The authors are grateful for the guidance provided by the United States Government Palliative Care Technical Working Group and to the Kenyan and Ugandan Country Teams. Finally we are grateful to the staff and patients at the participating facilities.

\section{Author details}

'King's College London, Department of Palliative Care, Policy \& Rehabilitation, Cicely Saunders Institute, Bessemer Road, London SE5 9RJ, UK. ${ }^{2}$ London School of Hygiene and Tropical Medicine, Keppel St, WC1E 7HT London, UK. ${ }^{3}$ African Palliative Care Association, Plot 850 Dr Gibbons Road, KampalaPO BOX 72518, Kampala, Uganda. ${ }^{4}$ Global Health and Knowledge Management Consultant, London, UK. ${ }^{5}$ Formerly APCA and Currently in USAID|ASSIST, University Research Company, Nairobi, Kenya. ${ }^{6}$ Futures Group, MEASURE Evaluation, University of North Carolina, Chapel Hill, USA.

Received: 4 August 2014 Accepted: 4 November 2014

Published online: 18 November 2014

\section{References}

1. Sherr L, Lampe F, Fisher M, Arthur G, Anderson J, Zetler S, Johnson M, Edwards S, Harding R: Suicidal ideation in UK HIV clinic attenders. AIDS 2008, 22(13):1651-1658.

2. Harding R, Clucas C, Lampe FC, Date HL, Fisher M, Johnson M, Edwards S, Anderson J, Sherr $L$ : What factors are associated with patient selfreported health status among HIV outpatients? A multi-centre UK study of biomedical and psychosocial factors. AIDS Care 2012, 24(8):963-971.

3. Harding R, Lampe FC, Norwood S, Date HL, Clucas C, Fisher M, Johnson M, Edwards S, Anderson J, Sherr L: Symptoms are highly prevalent among HIV outpatients and associated with poor adherence and unprotected sexual intercourse. Sex Transm Infect 2010, 86(7):520-524
4. Lampe FC, Harding R, Smith CJ, Phillips AN, Johnson M, Sherr L: Physical and psychological symptoms and risk of virologic rebound among patients with virologic suppression on antiretroviral therapy. J Acquir Immune Defic Syndr 2010, 54(5):500-505.

5. Harding R, Selman L, Agupio G, Dinat N, Downing J, Gwyther L, Mashao T, Mmoledi K, Moll T, Sebuyira LM, Ikin B, Higginson IJ: Prevalence, burden, and correlates of physical and psychological symptoms among HIV palliative care patients in sub-Saharan Africa: an international multicenter study. J Pain Symptom Manage 2012, 44(1):1-9.

6. Wakeham K, Harding R, Bamukama-Namakoola D, Levin J, Kissa J, ParkesRatanshi R, Muzaaya G, Grosskurth H, Lalloo DG: Symptom burden in HIV-infected adults at time of HIV diagnosis in rural Uganda. J Palliat Med 2010, 13(4):375-380.

7. World Health Organisation: Consitution of the World Health Organisation. 1948 [http://www.who.int/governance/eb/who_constitution_en.pdf]

8. World Health Organisation: Palliative Care. 2006 [http://www.who.int/hiv/ topics/palliative/care/en]

9. Simms VM, Higginson IJ, Harding R: What palliative care-related problems do patients experience at HIV diagnosis? A systematic review of the evidence. J Pain Symptom Manage 2011, 42(5):734-753.

10. Harding R, Easterbrook P, Dinat N, Higginson IJ: Pain and symptom control in HIV disease: under-researched and poorly managed. Clin Infect Dis 2005, 40(3):491-492.

11. Simms V, Higginson IJ, Harding R: Integration of palliative care throughout HIV disease. Lancet Infect Dis 2012, 12(7):571-575.

12. Harding R, Simms V, Krakauer E, Delima L, Downing J, Garanganga E, Green K, Gwyther L, Lohman D: Quality HIV care to the end of life. Clin Infect Dis 2011, 52(4):553-554. author reply 554

13. Dawson J, Doll H, Fitzpatrick R, Jenkinson C, Carr AJ: The routine use of patient reported outcome measures in healthcare settings. BMJ 2010, 340:c186.

14. Bendavid E, Bhattacharya J: The President's Emergency Plan for AIDS Relief in Africa: an evaluation of outcomes. Ann Intern Med 2009, 150(10):688-695.

15. Reynolds HW, Janowitz B, Wilcher R, Cates W: Contraception to prevent HIV-positive births: current contribution and potential cost savings in PEPFAR countries. Sex Transm Infect 2008, 84(Suppl 2):49-53.

16. Harding R, Simms V, Penfold S, McCrone P, Moreland S, Downing J, Powell RA, Mwangi-Powell F, Namisango E, Fayers P, Curtis S, Higginson IJ: Multi-centred mixed-methods PEPFAR HIV care \& support public health evaluation: study protocol. BMC Public Health 2010, 10:584.

17. Rutstein S, Johnson K: The DHS Wealth Index. DHS Comparative Reports. 2004 [http://www.measuredhs.com/pubs/pdf/CR6/CR6.pdf] MEASURE DHS+

18. Mast TC, Kigozi G, Wabwire-Mangen F, Black R, Sewankambo N, Serwadda D, Gray R, Wawer M, Wu AW: Measuring quality of life among HIV-infected women using a culturally adapted questionnaire in Rakai district, Uganda. AIDS Care 2004, 16(1):81-94.

19. Wu AW, Revicki DA, Jacobson D, Malitz FE: Evidence for reliability, validity and usefulness of the Medical Outcomes Study HIV Health Survey (MOS-HIV). Qual Life Res 1997, 6(6):481-493.

20. Wu AW, Hanson KA, Harding G, Haider S, Tawadrous M, Khachatryan A, Pashos CL, Simpson KN: Responsiveness of the MOS-HIV and EQ-5D in HIV-infected adults receiving antiretroviral therapies. Health Qual Life Outcomes 2013, 11:42.

21. Harding R, Selman L, Agupio G, Dinat N, Downing J, Gwyther L, Mashao T, Mmoledi K, Moll T, Sebuyira LM, Panjatovic B, Higginson IJ: Validation of a core outcome measure for palliative care in Africa: the APCA African Palliative Outcome Scale. Health Qual Life Outcomes 2010, 8:10.

22. Oken MM, Creech RH, Tormey DC, Horton J, Davis TE, McFadden ET, Carbone PP: Toxicity and response criteria of the Eastern Cooperative Oncology Group. Am J Clin Oncol 1982, 5(6):649-655.

23. Revicki DA, Sorensen S, Wu AW: Reliability and validity of physical and mental health summary scores from the Medical Outcomes Study HIV Health Survey. Med Care 1998, 36(2):126-137.

24. Gwatkin DR, Rustein S, Johnson K, Pande RP, Wagstaff A: Socioeconomic Differences in Health, Nutrition, and Population in Ghana. Country Reports on HNP and Poverty. Washington DC, USA: World Bank; 2000.

25. Selman L, Harding R, Higginson I, Gysels M, Speck P, EncompassCollaborative: Spiritual wellbeing in sub-Saharan Africa: the meaning and prevalence of 'feeling at peace'. BMJ Support Palliat Care 2011, 1(Suppl 1):A22. 
26. Selman L, Simms V, Penfold S, Powell RA, Mwangi-Powell F, Downing J, Gikaara N, Munene G, Higginson IJ, Harding R: 'My dreams are shuttered down and it hurts lots'-a qualitative study of palliative care needs and their management by HIV outpatient services in Kenya and Uganda. BMC Palliat Care 2013, 12(1):35.

27. Simms V, Gikaara N, Munene G, Atieno M, Kataike J, Nsubuga C, Banga G, Namisango E, Penfold S, Fayers P, Powell RA, Higginson IJ, Harding R: Multidimensional patient-reported problems within two weeks of HIV diagnosis in East Africa: a multicentre observational study. PLoS One 2013, 8(2):e57203.

28. Palermo T, Rawat R, Weiser SD, Kadiyala S: Food access and diet quality are associated with quality of life outcomes among HIV-infected individuals in Uganda. PLoS One 2013, 8(4):e62353.

29. Degroote S, Vogelaers DP, Vermeir P, Mariman A, De Rick A, Van Der Gucht B, Pelgrom J, Van Wanzeele F, Verhofstede C, Vandijck DM: Socio-economic, behavioural, (neuro)psychological and clinical determinants of HRQoL in people living with HIV in Belgium: a pilot study. J Int AIDS Soc 2013, 16:18643.

30. Harding R, Simms V, Penfold S, Downing J, Powell RA, Mwangi-Powell F, Namisango E, Moreland S, Gikaara N, Atieno M, Kataike J, Nsubuga C, Munene G, Banga G, Higginson IJ: The presence of CD4 counts for the management of HIV patients in East Africa: a multicentred study. AIDS Care 2013, 26(5):613-618.

31. Lowther K, Simms V, Selman L, Sherr L, Gwyther L, Kariuki H, Ahmed A, Ali Z, Jenkins R, Higginson IJ, Harding R: Treatment outcomes in palliative care: the TOPCare study. A mixed methods phase III randomised controlled trial to assess the effectiveness of a nurse-led palliative care intervention for HIV positive patients on antiretroviral therapy. BMC Infect Dis 2012, 12:288.

doi:10.1186/s12879-014-0613-1

Cite this article as: Harding et al:: Quality of life and wellbeing among HIV outpatients in East Africa: a multicentre observational study. BMC Infectious Diseases 2014 14:613.

\section{Submit your next manuscript to BioMed Central and take full advantage of:}

- Convenient online submission

- Thorough peer review

- No space constraints or color figure charges

- Immediate publication on acceptance

- Inclusion in PubMed, CAS, Scopus and Google Scholar

- Research which is freely available for redistribution 